Emily L. Johnson, MD Yi-Ting Chang, MSc Barbara Davit, PhD, JD Barry E. Gidal, PharmD Gregory L. Krauss, MD

Correspondence to Dr. Johnson: ejohns92@jhmi.edu

Editorial, page 1572

Supplemental data at Neurology.org

\section{Assessing bioequivalence of generic modified-release antiepileptic drugs}

Pît

\section{ABSTRACT}

Objectives: The purpose of this study was to determine how closely generic modified-release antiepileptic drugs (MR-AEDs) resemble reference (brand) formulations by comparing peak concentrations (Cmax), total absorption (area under the curve [AUC]), time to Cmax (Tmax), intersubject variability, and food effects between generic and reference products.

Methods: We tabulated Cmax and AUC data from the bioequivalence (BE) studies used to support the approvals of generic Food and Drug Administration-approved MR-AEDs. We compared differences in 90\% confidence intervals of the generic/reference AUC and Cmax geometric mean ratios, and intersubject variability, Tmax and delivery profiles and food effects.

Results: Forty-two MR-AED formulations were studied in 3,175 healthy participants without epilepsy in $97 \mathrm{BE}$ studies. BE ratios for AUC and Cmax were similar between most generic and reference products: $A \cup C$ ratios varied by $>15 \%$ in $11.4 \%$ of BE studies; Cmax varied by $>15 \%$ in $25.8 \%$ of studies. Tmax was more variable, with $>30 \%$ difference in 13 studies (usually delayed in the fed compared to fasting BE studies). Generic and reference MR products had similar intersubject variability. Immediate-release AEDs showed less intersubject variability in AUC than did MR-AEDs.

Conclusions: Most generic and reference MR-AEDs have similar AUC and Cmax values. Ratios for some products, however, are near acceptance limits and Tmax values may vary. Food effects are common with MR-AED products. High variability in pharmacokinetic values for once-a-day MR-AEDs suggests their major advantage compared to immediate-release AED formulations may be the convenience of less frequent dosing to improve adherence. Neurology ${ }^{\circledR} 2016 ; 86: 1597-1604$

\section{GLOSSARY}

AED = antiepileptic drug; ANDA = Abbreviated New Drug Application; $\mathbf{A U C}=$ area under the curve; $\mathbf{B E}=$ bioequivalence; $\mathbf{C B Z}=$ carbamazepine; $\mathbf{C l}=$ confidence interval; $\mathbf{C m a x}=$ maximum concentration; EMA = European Medicines Agency; FDA = Food and Drug Administration; GMR = geometric mean ratio; IR = immediate release; LEV = levetiracetam; $\mathbf{L T G}=$ lamotrigine; MR = modified release; PHT = phenytoin; PK = pharmacokinetic; Tmax = time to maximum concentration.

In the past 2 decades, many modified-release (MR) formulations of antiepileptic drugs (AEDs) have become available. MR-AEDs have advantages over immediate-release (IR) formulations in improving adherence ${ }^{1,2}$ and could potentially improve tolerability ${ }^{3,4}$ and seizure control. 4,5

Generic MR-AEDs are approved by the Food and Drug Administration (FDA) through an Abbreviated New Drug Application (ANDA) process in which formulations must meet bioanalytical and bioequivalence (BE) standards. ${ }^{6}$ European Medicines Agency (EMA) requirements are similar. ${ }^{7}$ Generic and reference formulations are compared in single-dose, crossover pharmacokinetic (PK) studies in healthy volunteers. Generic MR-AEDs qualify as bioequivalent if they provide maximum concentrations (Cmax) and total drug exposures (area under the curve $[A U C]$ ) that do not differ significantly from those of the branded reference formulation, ${ }^{6}$ defined as the $90 \%$ confidence interval (CI) for AUC and Cmax ratios for genericl reference products being within an acceptance range of $80 \%$ to $125 \%{ }^{6,7}$ How closely approved generic MR-AEDs resemble branded reference formulations in these BE studies

From the Department of Neurology (E.L.J., G.L.K.), The Johns Hopkins University School of Medicine, Baltimore, MD; Johnson \& Johnson (Y.-T.C.), New Brunswick; Merck \& Co., Inc. (B.D.), Kenilworth, NJ; and University of Wisconsin (B.E.G.), School of Pharmacy, Madison, WI. Go to Neurology.org for full disclosures. Funding information and disclosures deemed relevant by the authors, if any, are provided at the end of the article. 
has not been reported; i.e., are ratios for most products nearer to one, or are they near acceptance limits? The slow-release technologies of MR-AEDs introduce an additional factor into BE assessment: MR-AEDs could potentially have similar Cmax and AUC but varying concentration-time profiles and time to Cmax (Tmax).

Some clinicians and patients have reported that switches between reference and generic AED products are associated with seizures and adverse drug effects. ${ }^{8-10}$ We evaluated how closely generic MR-AED formulations resemble reference formulations in the ANDA BE studies, compared variability of AED concentrations across participants, and determined whether some generic and reference products have disparate delivery profiles and Tmax values.

METHODS BE study data. We obtained average BE and demographic data from the FDA Center for Drug Evaluation and Research, Office of Generic Drugs, for generic MR-AED formulations approved through May 2014. ANDAs for most generic formulations contained results of separate fasting and fed $\mathrm{BE}$ studies. We tabulated the geometric mean ratios (GMRs) and their 90\% CIs for the area under the plasma concentration curve (AUC) calculated to the last measured concentration $\left(\mathrm{AUC}_{0-\mathrm{t}}\right)$ and extrapolated to infinity $\left(\mathrm{AUC}_{0-\infty}\right)$; the maximum plasma concentration (Cmax); and the coefficient of variation for $\mathrm{AUC}_{0-\mathrm{t}}, \mathrm{Cmax}$, and Tmax. Demographic information consisted of age (mean and range), sex, ethnicity, and body mass index.

The available generic MR-AEDs formulations included phenytoin (PHT) (8 products); carbamazepine (CBZ) (5 products); divalproex sodium (7 products); levetiracetam (LEV) (15 products); and lamotrigine (LTG) (7 products). ${ }^{11}$

Analysis of generic and reference MR-AED PK data. We classified the $90 \%$ CI limit of AUC and Cmax GMRs as the maximum of the absolute value of the upper or lower $90 \%$ $\mathrm{CI}$ subtracted from 1.00 , displayed as a percentage. For example, a BE study with $90 \%$ CI of $0.93-1.04$ had a $90 \%$ CI limit of $7 \%$. We classified these differences in AUC and Cmax ratios in $5 \%$ bands for the acceptance ranges of $80 \%$ to $125 \%$ and determined the proportion of BE studies in each band. We used the same methods to assess food effects by comparing fasting and fed BE studies. We compared intersubject variability in AUC and Cmax for test and reference products by calculating intersubject SDs for AUC and Cmax for each BE study and screening for major (>50\%) differences in SDs in the PK values. We compared generic/reference Tmax ratios and identified formulations with large $(>20 \%)$ differences in Tmax. We visually identified reference and test MR products whose PK concentration-time profiles had large differences in both Tmax and in concentration slopes.

Comparison of MR-AED to IR-AED variability. We compared intersubject variability in AUC and Cmax between IR- and MR-AED products, using previously analyzed IR-AED ANDA data. ${ }^{12}$ We categorized the coefficient of variation of AUC and Cmax for the BE studies into $10 \%$ bands and compared proportions of formulations in each band for the IR and MR products.

RESULTS Participants. Forty-two generic MR-AED products were evaluated in $97 \mathrm{BE}$ studies, enrolling 3,175 healthy participants. Demographic data were available for 93 studies. Participants were predominantly male $(86.6 \%)$, with a mean age of 29.7 years (range, 18-79). Ethnicities reflected the locations of study sites and were as follows: Asian 47.27\%, Caucasian 35.37\%, black 12.80\%, Hispanic $2.41 \%$, and other $2.12 \%$. Participant demographic factors were unequally distributed in many studies; $51.6 \%$ enrolled Asians only and $51.6 \%$ enrolled males only. The mean body mass index (available for 2,560 participants) was 23.52 $\mathrm{kg} / \mathrm{m}^{2}$ (range, 14.3-32.7).

MR technologies. MR-AEDs are produced using many drug-delivery technologies, including coated matrices, lipid matrices, matrix pores, coated micro-granules, osmotic pumps, reservoir systems, multiphasic beads, and osmotic shells. ${ }^{13,14}$ Manufacturers of generic MR products are permitted to use different delivery technologies than reference products, and these often varied between generic and reference AED formulations. For example, MR-LEV formulations used matrix dissolving tablets, osmotic pumps, and matrix pore delivery technologies. Manufacturers are not required to report release technologies for formulations and these were not systematically available for comparison.

BE of generic and reference MR-AEDs. Total drug delivery $\left(\mathrm{AUC}_{0-\mathrm{t}}\right)$ for generic and reference MR-AED formulations was very similar for most products (figure 1 and table e-1 on the Neurology ${ }^{\circledR}$ Web site at Neurology.org): the ratios for $\mathrm{AUC}_{0-\mathrm{t}}$ (maximum upper or lower $90 \%$ CI limit) varied by $<15 \%$ for the large majority $(88.6 \%)$ of studies; $30 \%$ of MRAED products differed by $<5 \%$. The ratios between reference and generic product $\mathrm{AUC}_{0-\mathrm{t}}$ maximum $90 \%$ CI limits were greater than $15 \%$ in 11 studies. The GMR point estimates for AUC fell within 10\% of the reference point estimates in nearly all $\mathrm{BE}$ studies (97.9\%). Even though all approved products met ANDA acceptance standards, the $\mathrm{AUC}_{0-\mathrm{t}}$ ratios differed substantially for one-fifth (22.7\%) of reference and generic formulations (i.e., the $90 \%$ CI for the AUC ratios did not include 1.00) (figure 2, $A$ and $B)$.

The peak drug concentrations (Cmax) varied somewhat more between generic and reference formulations than did $\mathrm{AUC}_{0-\mathrm{t}}$. For the majority (74.2\%) of BE studies, Cmax ratios between generic and reference MR-AED products differed by $<15 \%$ 


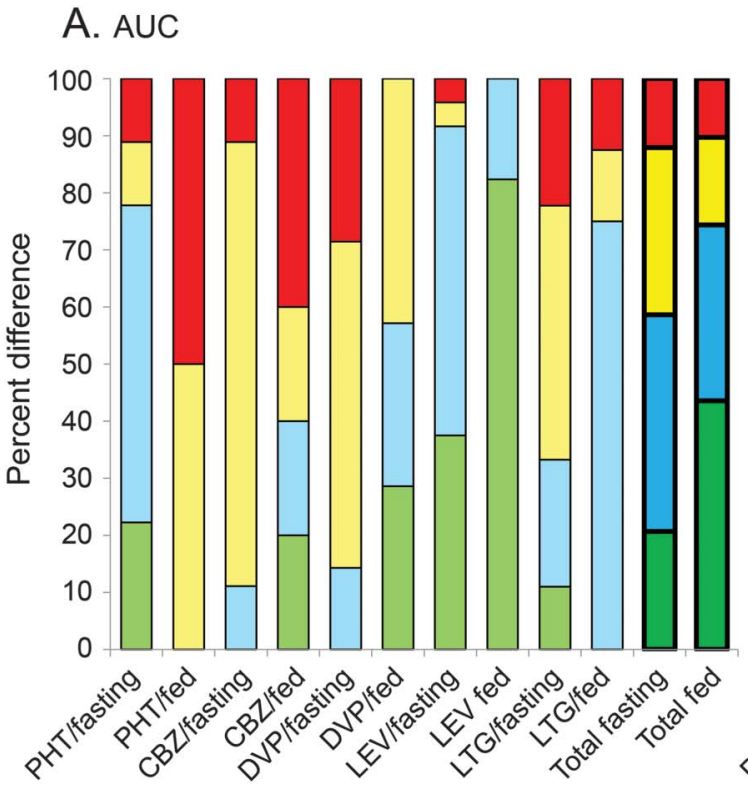
B. Cmax

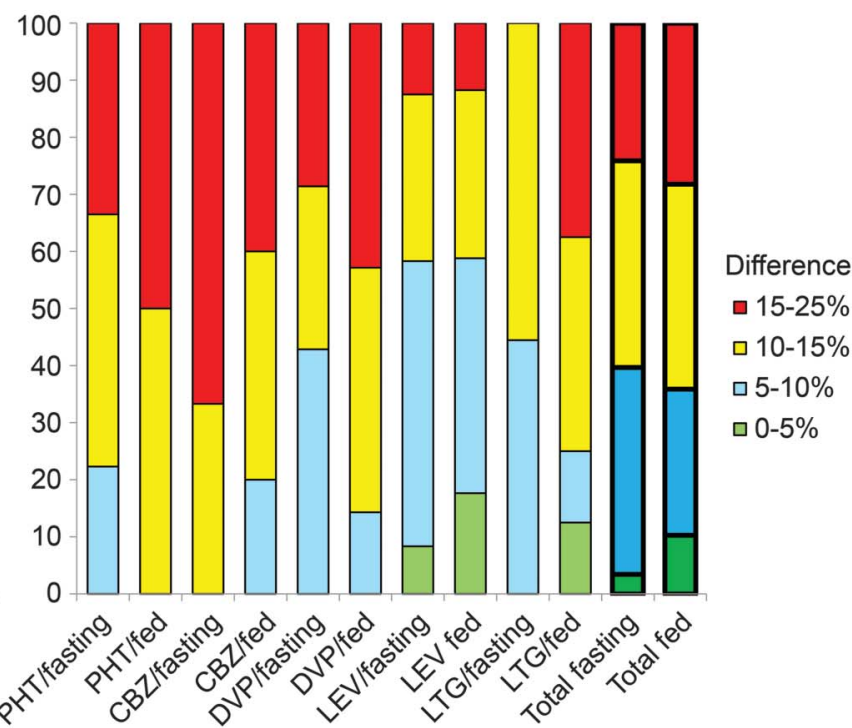

Differences in 90\% confidence interval limits of total drug exposure (AUC) (A) and Cmax (B) geometric mean ratios for generic and reference modifiedrelease antiepileptic drug formulations, classified in $5 \%$ increments. Percent of bioequivalence study results in each category are shown on the vertical axis. The majority of $\mathrm{AUC}$ and $\mathrm{Cmax}$ values differ by $<15 \%$. AUC = area under the curve; $\mathrm{CBZ}=$ carbamazepine; $\mathrm{Cmax}=$ maximum concentration; $\mathrm{DVP}=$ divalproex; LEV = levetiracetam; LTG = lamotrigine; $\mathrm{PHT}=$ phenytoin.

(maximum upper or lower $90 \%$ CI limits). The GMR point estimates for Cmax for generic and reference products varied by less than $10 \%$ in 76 of 97 studies (78.3\%). The Cmax differed substantially between reference and generic test products for approximately half $(51.6 \%)$ of $\mathrm{BE}$ studies (i.e., the 90\% CI limits for Cmax did not include 1.00) (figure 2, C and D).

Medications varied in their AUC and Cmax ratios: nearly all LEV MR products (95\%) had AUC ratio limits of $<10 \%$, and the majority of LEV products (59\%) had Cmax ratio limits of $<10 \%$. AUC ratio limits were relatively small for most drugs: $\mathrm{CBZ}$ was the most variable and only one-fifth (21\%) had AUC ratio limits of $>15 \%$. CBZ Cmax ratio limits were high compared to other drug formulations: the majority (57\%) of CBZ products had Cmax ratio limits $>15 \%$.

The distribution of AUC and Cmax for MRAED products was not broad; the range between the upper and lower $90 \%$ CIs was greater than $20 \%$ in only a small minority $(6.2 \%)$ of studies for AUC and Cmax.

$\mathrm{BE}$ studies in the fed state showed less variation in the AUC between generic and reference products than did fasting studies; nearly half (43.6\%) of fed studies had generic AUC 90\% CI limit within 5\% of reference, vs only one-fifth (20.7\%) of fasting studies with $90 \%$ CI limit within $5 \%$ of reference. Fewer Cmax studies had such low variation; only $10.2 \%$ of fed and 3.5\% of fasting Cmax studies had a very low (<5\%) $90 \%$ CI limit.

Variability in AUC and Cmax for reference compared to generic MR-AED formulations. The variability in the distributions of $\mathrm{PK}$ values for generic and reference products for participants in each BE study was similar across the studies: no studies had $>50 \%$ difference between the test/reference ratio for $\mathrm{SD}$ for $\mathrm{AUC}_{0-\mathrm{t}}$ (figure e-1). SDs for Cmax varied by $>50 \%$ between generic and reference for 4 studies (4\%, one each in LTG and LEV under fed conditions, and CBZ and PHT under fasting conditions).

Comparison of concentration profiles and Tmax for generic and reference MR-AED formulations. Tmax varied considerably across different drugs, with median Tmax for PHT less than 10 hours in all 11 studies, while the median CBZ Tmax was at least 25 hours in half of the studies. The Tmax ratio for most reference and generic products was between 0.8 and 1.2 and differed by $>20 \%$ in only $17(17.5 \%)$ of $\mathrm{BE}$ studies (figure 3). A small group of generic products, however, had Tmax ratios that varied markedly from reference products, with Tmax ratios ranging from 0.38 (LTG, fed) to 1.71 (also LTG, fed). In this group, test and reference Tmax values differed by up to 15 hours and concentration-vs-time profiles appeared dissimilar (figure 4); at some time points, concentrations differed by more than $50 \%$ (figure $4 \mathrm{~B}$; i.e., 10 hours postdose). 
A

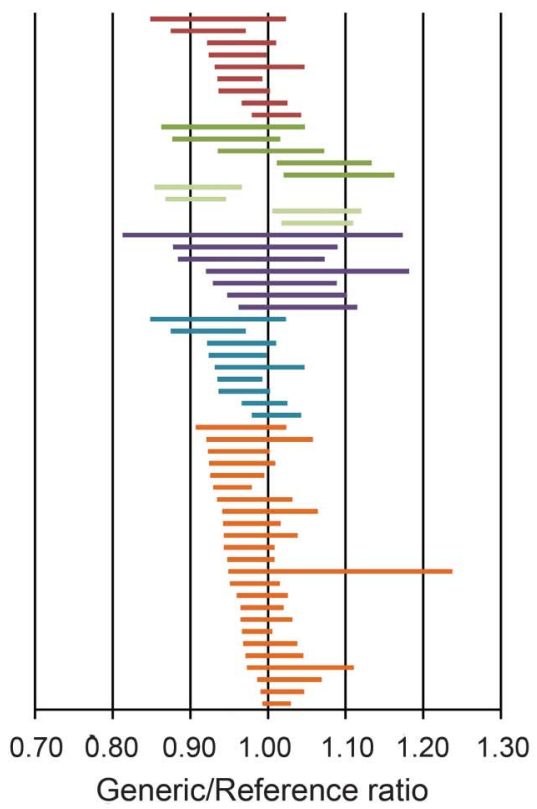

C

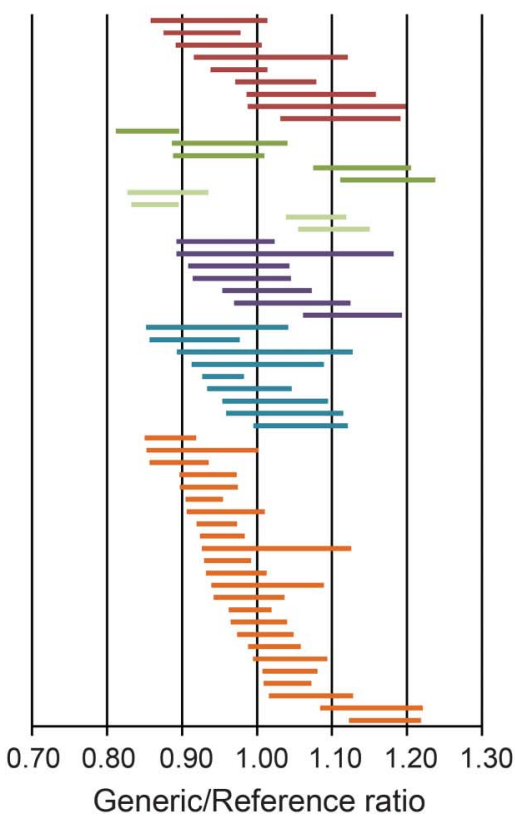

B

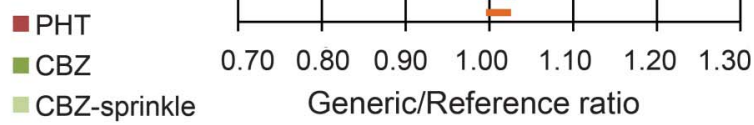

口DVP

$\square$ LTG

- LEV
D

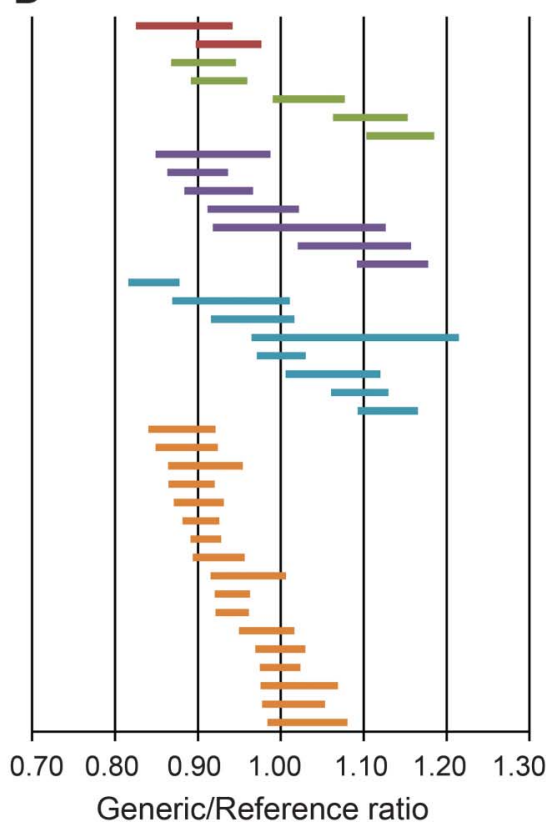

Ninety percent confidence intervals of generic/reference ratios for each product: total drug exposure (AUC) (A and B) and Cmax (C and D) under fed and fasting conditions ([A] AUC under fasting conditions; [B] AUC under fed conditions; [C] Cmax under fasting conditions; and [D] Cmax under fed conditions). The majority of confidence intervals include 1.00. AUC = area under the curve; CBZ = carbamazepine; Cmax = maximum concentration; DVP = divalproex; LEV = levetiracetam; LTG = lamotrigine; $\mathrm{PHT}=$ phenytoin.

A small number of studies had Cmax near acceptance limits in conjunction with disparate Tmax; 5 of 97 studies (5.2\%) had a Cmax CI limit of $>15 \%$ as well as a Tmax difference of $>20 \%$. Two studies were LTG in the fed condition, 2 were LEV in the fasting condition, and one PHT in the fasting condition.

Food effects for generic vs reference formulations. Separate fed and fasting BE studies evaluated food effects in
$76 \mathrm{BE}$ studies (38 sets of reference/generic formulations). Most products (generic and reference) had similar Cmax and AUC in the fed and fasting states (figure e-2). These differed, however, for 22 of 76 (28.9\%) generic and reference studies, with Cmax often increased during the fed compared to the fasting state. Fourteen of $22(63.6 \%)$ generic and reference CBZ and divalproex formulations had $>20 \%$ increase in Cmax in the fed compared to the fasting state. 


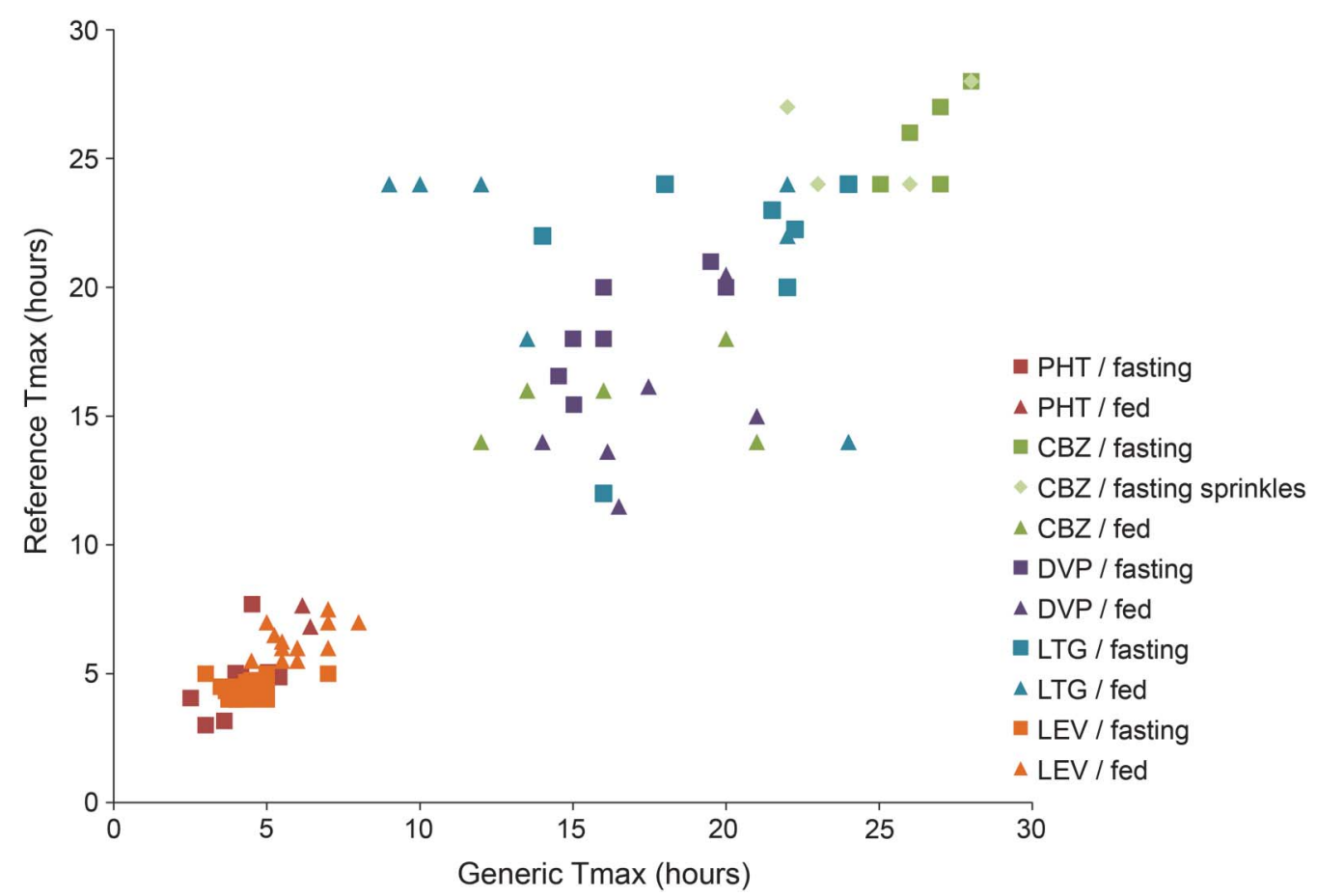

Comparison of arithmetic mean Tmax for generic and reference antiepileptic drug formulations in bioequivalence studies. The Tmax arithmetic means were similar between generic and reference formulations in most cases; however, 5 of 8 LTG studies in the fed state had Tmax differences of $>20 \%$. CBZ = carbamazepine; DVP = divalproex; LEV = levetiracetam; LTG = lamotrigine; PHT = phenytoin; Tmax = time to maximum concentration.

In contrast to Cmax and AUC, Tmax varied markedly between fed and fasting studies in twothirds of BE studies (both generic and reference products): Tmax was $>20 \%$ longer in fed studies compared to fasting studies in 18 of 38 generic and 13 of 38 reference products (figure e-2C). Food effects on Tmax were particularly common for MRLEV and MR-LTG products.

Figure 4 Example concentration-time profiles from a single set of divalproex and a single set of lamotrigine generic and reference products
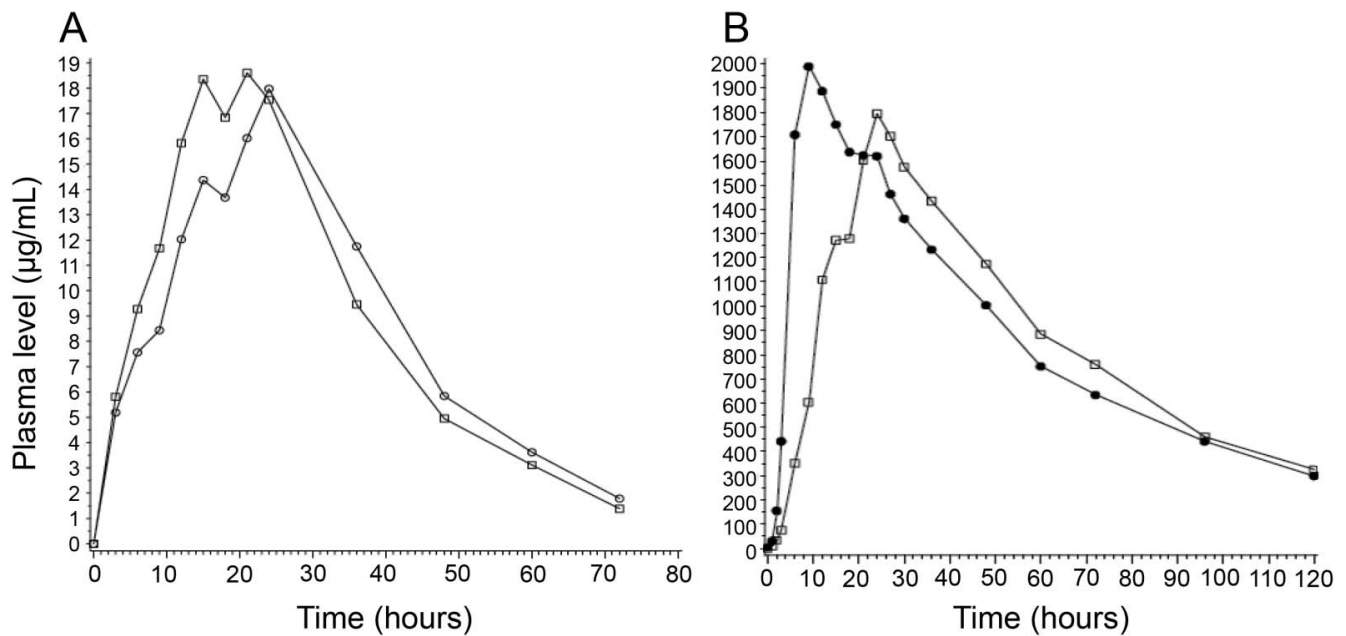

(A) Divalproex generic (circles) vs reference (squares) product concentration-time profiles. The double-peaked pharmacokinetic profile of the reference product is also present in the generic product. Tmax ratio $=1.4$. (B) Lamotrigine generic (circles) and reference (squares) product concentration-time profiles. From 10 hours through 15 hours, plasma level is up to $50 \%$ different, although AUC and Cmax are within acceptable limits. Tmax ratio $=0.37$. AUC $=$ area under the curve; $\mathrm{Cmax}$ $=$ maximum concentration; $T \max =$ time to maximum concentration. 
Comparison of MR-AED to IR-AED intersubject variability. Fasting BE studies of both $M R$ and IR formulations of LTG, LEV, and CBZ products were available. The reference and generic formulations had similar intersubject variability in each individual IR and MR product study. When all reference and generic MR products were compared to all reference and generic IR products, however, the IR products (AUC) had lower variability in PK measures for participants compared to MR products (table 1). Most of the IR-LEV products (92.3\%) had $<20 \%$ intersubject variability for AUC, compared to 64.6\% of MR-LEV products. CBZ also had lower AUC variability in IR compared to MR products (64.3\% of IR vs $37.5 \%$ of MR products had $<20 \%$ variability). LTG had higher variability in AUC than did LEV or CBZ: only $4 \%$ of IR-LTG products had variability $<20 \%$ (while no MR-LTG products had $<20 \%$ variance); the majority of LTG products had AUC variability of $20 \%$ to $30 \%$ (56\% of IR-LTG products) or $30 \%$ to $40 \%$ (55.6\% of MR-LTG products).

Cmax variability was lower in IR compared to MR products for every drug except CBZ; more than half of IR-LTG products had $<20 \%$ variability, while only $38.9 \%$ of MR products had $<20 \%$ variability (table 1 ).

DISCUSSION Although the objective of ANDA BE studies is to determine whether generic formulations meet regulatory standards, comparing BE data from a large number of these small, blinded crossover studies can provide valuable information about how closely generic MR-AEDs copy brand-name products. Intersubject variability of drug concentrations for MR-AED products also may provide estimates of the products' performance in clinical use. The majority of MR-AED formulations had comparable AUC and Cmax values. A small number of products, however, had PK ratios near acceptance limits, with the AUC of CBZ being close to acceptance limits in one-fifth of cases. The variability in BE studies across participants was similar between generic and reference products, suggesting either generic or reference could be used in initial treatment.

This study has several limitations. First, BE studies used in this analysis are all single-dose crossover studies in patients without epilepsy, are powered to meet FDA acceptance standards, and do not study tolerability and efficacy of AEDs. Second, no participant-level information was available for more detailed analysis. In addition, while tablet components are listed in approved drug product labeling, MR technologies are considered proprietary and were not released for many products. This prevented a comprehensive examination of the relationship between types of MR technologies and BE differences. Patients are often concerned when tablet ghost shells pass into their stools, which is occasionally reported by our patients; most current labeling for matrix pore, osmotic shell, and other AED products that may appear in the stool do not carry information for patients about ghost shells. This occurs with Novartis Tegretol XR (which carries a warning in the product insert) and with the Teva brand of LEV XR (which does not).

\begin{tabular}{|c|c|c|c|c|c|c|c|c|}
\hline \multirow[t]{3}{*}{ Table 1} & \multicolumn{8}{|c|}{ Intersubject variability of MR-AEDs vs IR-AEDs } \\
\hline & \multicolumn{2}{|l|}{ LTG } & \multicolumn{2}{|l|}{ LEV } & \multicolumn{2}{|l|}{ CBZ } & \multirow[b]{2}{*}{ Total MR } & \multirow[b]{2}{*}{ Total IR } \\
\hline & MR & IR & MR & IR & MR & IR & & \\
\hline \multicolumn{9}{|l|}{ AUC CV, \% } \\
\hline$<20$ & $0(0)$ & $2(4)$ & $31(64.6)$ & 24 (92.3) & $6(37.5)$ & $18(64.3)$ & $37(45.1)$ & $44(42.3)$ \\
\hline $20-30$ & $8(44.4)$ & $28(56)$ & $17(35.4)$ & $2(7.7)$ & $10(62.5)$ & 8 (28.6) & $35(42.7)$ & 38 (36.5) \\
\hline $30-40$ & $10(55.6)$ & 19 (38) & $0(0)$ & $0(0)$ & $0(0)$ & $2(7.1)$ & 10 (12.2) & 21 (20.2) \\
\hline$>40$ & $0(0)$ & $1(2)$ & $0(0)$ & $0(0)$ & $0(0)$ & $0(0)$ & $0(0)$ & $1(1.0)$ \\
\hline Total studies & 18 & 50 & 48 & 26 & 16 & 28 & 82 & 104 \\
\hline \multicolumn{9}{|l|}{$\mathrm{Cmax}_{\mathrm{CV}}$ \% } \\
\hline$<20$ & 7 (38.9) & $27(54)$ & 21 (43.7) & $12(46.2)$ & 15 (93.7) & 24 (85.7) & $43(52.4)$ & $63(60.6)$ \\
\hline $20-30$ & $10(55.6)$ & $23(46)$ & $26(54.2)$ & $14(53.8)$ & $1(6.3)$ & $2(7.1)$ & 37 (45.1) & 39 (37.5) \\
\hline $30-40$ & $1(5.6)$ & $0(0)$ & $1(2.1)$ & $0(0)$ & $0(0)$ & $2(7.1)$ & $2(2.4)$ & 2 (1.9) \\
\hline$>40$ & $0(0)$ & $0(0)$ & $0(0)$ & $0(0)$ & $0(0)$ & $0(0)$ & $0(0)$ & $0(0)$ \\
\hline Total studies & 18 & 50 & 48 & 26 & 16 & 28 & 82 & 104 \\
\hline
\end{tabular}

Abbreviations: $\mathrm{AED}=$ antiepileptic drug; $\mathrm{AUC}=$ area under the curve (total concentration); $\mathrm{CBZ}=$ carbamazepine; $\mathrm{Cmax}=$ maximum concentration; $\mathrm{CV}=$ coefficient of variation; IR = immediate release; LEV = levetiracetam; LTG = lamotrigine; MR = modified release.

Data are presented as no. of studies (\%) in each category of variance. 
Our finding that many MR products (generic and reference formulations) had substantial PK differences in the fed BE studies compared to fasting studies is consistent with prior findings in other MR products. ${ }^{15,16}$ Food administration often slows gastric transit time and increases $\mathrm{pH}$, which may contribute to delayed Tmax; the effects vary depending on the relevant MR mechanism and the individual drug characteristics, with high interindividual variability. ${ }^{17,18}$ Patients should be counseled to take MR-AEDs consistently in either the fed or fasting state. Cmax values for CBZ and divalproex, and Tmax for LEV and LTG MR products, were particularly affected by food.

Many reference-generic switches and genericgeneric switches are possible. Recent crossover studies of IR-LTG products show that reference-generic ${ }^{19}$ and disparate generic-generic ${ }^{20}$ switches provide comparable PK parameters at steady state. These crossover LTG studies found substantial individual variability in absorption: approximately $15 \%$ of participants had $>20 \%$ intrasubject variability with reexposure to the same product, either reference or generic. ${ }^{19}$ All MR products have intrinsic variability in once-a-day drug delivery. Although not studied directly, the varying Tmax values across generic products may support trying to minimize formulation switches between generic MR products.

Cmax and AUC had broader distributions for MR-AEDs compared to IR-AED products: the range between the upper and lower 90\% CI for Cmax and AUC ratios were larger for MR-AEDs compared to IR-AEDs. ${ }^{12}$ This may be attributable to the fact that most once-a-day MR-AED formulations are more complex than formulations for IR-AED products or even twice-a-day MR products. Most ANDA submissions include in vitro dissolution studies; the FDA uses dissolution studies to assess quality, and sometimes as a surrogate for in vivo BE studies of $\mathrm{MR}$ products, which require more complex compartment modeling of kinetics than IR products. ${ }^{6}$

Tmax varied and was sometimes similar to IR products; however, the fluctuation index (difference in peak to trough concentration compared to the average concentration) is lower in MR products, which may provide clinical benefits such as improved tolerability of $\mathrm{MR}$ compared to IR products. Although the Tmax of MR-CBZ was $>24$ hours in the fed state for all products studied, their twice-daily administration reduces the fluctuation index, which may contribute to their observed improved tolerability compared to IR-CBZ.,

Despite the lower fluctuation index, MR-AEDs had higher intersubject variability compared to IRAED products. Intersubject variability of AUC was $<30 \%$ for the majority of both MR- and IR-CBZ and MR- and IR-LEV; LTG intersubject variability was $>30 \%$ in more than half of MR-LTG products (generic and reference), and $>30 \%$ in $40 \%$ of IRLTG products. In patients starting therapy with an MR-AED formulation, the higher intersubject variability may not be relevant. However, clinicians may wish to compare drug serum levels in some patients when switching from IR to MR products. These intersubject differences were especially large for IR-and MR-LTG formulations.

Clinicians have speculated that occasional missed doses of a once-daily MR product may produce larger changes in concentration compared to a missed twicedaily IR dose. Modeling studies of oxcarbazepine and LTG, however, predicted similar trough levels after missed or delayed IR or MR doses. ${ }^{21,22}$ MR formulations appear to minimize effects of delayed doses, ${ }^{21,22}$ although steady-state concentrations took longer to recover after missed MR doses compared to IR doses. ${ }^{21}$

It is intrinsic to MR or "slow-release" technology to delay Tmax compared to IR products, and it is unclear whether major differences in Tmax between products would be clinically significant in the small subgroup of AED MR formulations with differing concentration profiles. Tmax, however, varied by up to 15 hours between several generic and brand products, and at some PK time points, drug concentrations differed by up to $50 \%$. Chronic dosing may ameliorate these differences at steady state, ${ }^{19}$ although the fluctuation index may be higher in formulations with higher Cmax. ${ }^{21}$ The FDA and EMA may reject MR products, including AEDs, with disparate Tmax and delivery profiles based on a clinical review. ${ }^{23}$ For example, the FDA recently withdrew approval of time-release methylphenidate products that provided low concentrations at critical time points for effectiveness. The FDA now requires that partial AUC measurements be comparable for new generic test and reference MR methylphenidate products for AUC between 0-3 hours, 3-7 hours, and 7-12 hours (in the fasting state); partial AUC for fed studies must be comparable between 0-4 hours, 4-8 hours, and 8-12 hours. ${ }^{24}$ The EMA once proposed applying partial AUC comparisons to BE standards for MR products but has not applied these to AEDs. ${ }^{25}$ Partial AUC may be a way to capture differences in concentration-time profiles that could lead to peak-related adverse events.

Our review of BE studies of approved generic MRAED formulations shows that most generic MR-AED products provide AED concentrations similar to reference MR products and have comparable intersubject variability despite using varying delivery technologies. Food often delays absorption of MRAED products, suggesting that patients should take these consistently in fasting or fed states. A small number of MR-AED products have Tmax and 
delivery profiles that differ from the reference products, and it is unclear whether these PK differences would be clinically significant. Most MR-AEDs have higher intersubject variability than IR-AEDs, and the main advantages of MR compared to IR products appear to be their potential to improve tolerability and to improve adherence.

\section{AUTHOR CONTRIBUTIONS}

Dr. Johnson interpreted the data and drafted the manuscript. Ms. Chang analyzed and interpreted the data. Dr. Davit revised the manuscript substantially for content. Dr. Gidal revised the manuscript substantially for content. Dr. Krauss designed the study, interpreted the data, and revised the manuscript substantially for content.

\section{STUDY FUNDING}

No targeted funding reported.

\section{DISCLOSURE}

E. Johnson, Y. Chang, and B. Davit report no disclosures relevant to the manuscript. B. Gidal receives honoraria from serving as a speaker for UCB and XenoPort and from consulting for Eisai and Upsher-Smith Labs. G. Krauss reports no disclosures relevant to the manuscript. Go to Neurology.org for full disclosures.

Received July 30, 2015. Accepted in final form December 28, 2015.

\section{REFERENCES}

1. Cramer J, Vachon L, Desforges C, Sussman NM. Dose frequency and dose interval compliance with multiple antiepileptic medications during a controlled clinical trial. Epilepsia 1995;36:1111-1117.

2. Cramer JA, Mattson RH, Prevey ML, Scheyer RD, Ouellette VL. How often is medication taken as prescribed? A novel assessment technique. JAMA 1989;261: 3273-3277.

3. Miller AD, Krauss GL, Hamzeh FM. Improved CNS tolerability following conversion from immediate- to extended-release carbamazepine. Acta Neurol Scand 2004;109:374-377.

4. Ficker DM, Privitera M, Krauss G, Kanner A, Moore JL, Glauser T. Improved tolerability and efficacy in epilepsy patients with extended-release carbamazepine. Neurology 2005;65:593-595.

5. Leppik IE, Hovinga CA. Extended-release antiepileptic drugs: a comparison of pharmacokinetic parameters relative to original immediate-release formulations. Epilepsia 2013;54:28-35.

6. Food and Drug Administration. Guidance for industry: SUPAC-MR: modified release solid oral dosage forms. Available at: http://www.fda.gov/downloads/Drugs/Guidances/ ucm070640.pdf. Accessed September 15, 2014.

7. European Medicines Agency. Guideline on the investigation of bioequivalence. Available at: http://www.ema.europa.eu/ docs/en_GB/document_library/Scientific_guideline/2010/ 01/WC500070039.pdf. Accessed May 28, 2015.

8. Berg MJ, Gross RA, Tomaszewski KJ, Zingaro WM, Haskins LS. Generic substitution in the treatment of epilepsy: case evidence of breakthrough seizures. Neurology 2008;71:525-530.

9. Burkhardt RT, Leppik IE, Blesi K, Scott S, Gapany SR, Cloyd JC. Lower phenytoin serum levels in persons switched from brand to generic phenytoin. Neurology 2004;63:1494-1496.
10. Nuwer MR, Browne TR, Dodson WE, et al. Generic substitutions for antiepileptic drugs. Neurology 1990;40: 1647-1651.

11. Food and Drug Administration. Orange Book: Approved Drug Products with Therapeutic Equivalence Evaluations. Available at: http://www.accessdata.fda.gov/scripts/cder/ ob/. Accessed July 27, 2015.

12. Krauss GL, Caffo B, Chang YT, Hendrix CW, Chuang K. Assessing bioequivalence of generic antiepilepsy drugs. Ann Neurol 2011;70:221-228.

13. Reed RC, Meinhold J, Dutta S, Liu W, Qiu Y. What do the suffixes_-XR, ER, chrono, chronosphere-really mean as it pertains to modified-release antiepileptic drugs? J Clin Pharm Ther 2010;35:373-383.

14. Nokhodchi A, Raja S, Patel P, Asare-Addo K. The role of oral controlled release matrix tablets in drug delivery systems. Bioimpacts 2012;2:175-187.

15. Wilder BJ, Leppik I, Hietpas TJ, Cloyd JC, Randinitis EJ, Cook J. Effect of food on absorption of Dilantin Kapseals and Mylan extended phenytoin sodium capsules. Neurology 2001;57:582-589.

16. Schug BS, Brendel E, Wolf D, Wonnemann M, Wargenau M, Blume HH. Formulation-dependent food effects demonstrated for nifedipine modified-release preparations marketed in the European Union. Eur J Pharm Sci 2002; 15:279-285.

17. Riethorst D, Mols R, Duchateau G, Tack J, Brouwers J, Augustijns P. Characterization of human duodenal fluids in fasted and fed state conditions. J Pharm Sci Epub 2015 Jul 30.

18. Augustijns P, Wuyts B, Hens B, Annaert P, Butler J, Brouwers J. A review of drug solubility in human intestinal fluids: implications for the prediction of oral absorption. Eur J Pharm Sci 2014;57:322-332.

19. Ting TY, Jiang W, Lionberger R, et al. Generic lamotrigine versus brand-name Lamictal bioequivalence in patients with epilepsy: a field test of the FDA bioequivalence standard. Epilepsia 2015;56:1415-1424.

20. Privitera M, Diaz F, Dworetzky B, et al. Bioequivalence testing of disparate generic lamotrigine products using chronic dosing in people with epilepsy: the EQUIGEN Study. Presented at the 2014 American Epilepsy Society meeting; December 6, 2014; Seattle. Abstract 1.324.

21. Chen C, Wright J, Gidal B, Messenheimer J. Assessing impact of real-world dosing irregularities with lamotrigine extended-release and immediate-release formulations by pharmacokinetic simulation. Ther Drug Monit 2013;35: 188-193.

22. Brittain ST, Wheless JW. Pharmacokinetic simulations of topiramate plasma concentrations following dosing irregularities with extended-release vs. immediate-release formulations. Epilepsy Behav 2015;52:31-36.

23. Davit B, Braddy AC, Conner DP, Yu LX. International guidelines for bioequivalence of systemically available orally administered generic drug products: a survey of similarities and differences. AAPS J 2013;15:974-990.

24. Food and Drug Administration. Draft guidance on methylphenidate hydrochloride. Available at: http://www.fda.gov/ downloads/drugs/guidancecomplianceregulatoryinformation/ guidances/ucm320007.pdf. Accessed July 27, 2015.

25. European Medicines Agency. Overview of comments received on "guideline on quality of oral modified release products.” Available at: http://www.ema.europa.eu/docs/ en_GB/document_library/Other/2014/07/WC500170466. pdf. Accessed July 27, 2015. 